\title{
On the Translation and Introduction Strategy of Taizhou Hehe Culture*
}

\author{
LI Chun-feng \\ Taizhou University, Linhai, China
}

\begin{abstract}
Taizhou Hehe Culture is a regional culture and core of Chinese Hehe Culture. On the circumstances of Chinese culture's going out to world, this paper analyzes the translation and introduction strategy of Taizhou Hehe Culture, so as to inherit and innovate Taizhou regional culture and further promote the cultural communication between China and other countries.
\end{abstract}

Keyword: Taizhou Hehe Culture, translation and introduction, foreignization, domestication

“Our ancestors created an unparalleled culture, and the Hehe (和合) culture is one of the essence of this culture”, Chairman Xi Jinping (2005, p. 8) said in the book Zhi Jiang Xin Yu. “和” refers to harmony, peace, etc., while “合” refers to convergence, integration, and union. This kind of tolerant character of harmony and moderation, understanding and tolerance, social morality, and harmony without uniformity is a cultural concept pursued by our nation. Strengthening Taizhou Hehe (和合) Culture translation work is not only in line with the "area" initiative and cultural background of the strategy of "going out", but sticks to Taizhou City orientation of constructing “a city with mountains, seas and water, a holy land with Hehe (和合) culture”, which plays an important role in inheriting and innovating the unique regional culture, enhancing Taizhou cultural soft power, facilitating Taizhou social and economic development, speeding up the maritime silk route into the 21st century, and constructing the powerful cultural city.

\section{Status Quo of Translation and Introduction of Taizhou Hehe (和合) Culture}

The domestic academic circles' attention to Hehe (和合) culture originated from the study of the philosophy of "harmony between man and nature", which began in the mid-1990s. After more than 20 years of development, a number of influential scholars and achievements emerged. Among them, Professor Zhang Liwen (张立文) from Renmin University of China published a monograph The Concept of Harmony - the Idea of Cultural Strategy in the 21st Century of 780,000 words in 1996, which established the theoretical system of harmony. Professor Zhang Dainian (张岱年), Professor Ji Xianlin (季羡林), and Professor Tang Yijie (汤一介) of Peking University, Master Qian Mu (钱穆) of traditional Chinese, and Mr. Cheng Siyuan (程思远), director of the organizing committee of "Chinese harmony culture promotion project" have all carried out fruitful researches on Chinese Hehe (和合) Culture.

Taizhou is not only the residence of “Two Saints of Hehe (和合)”, but also a typical area of Chinese Hehe (和合) Culture. Since the reform and opening up, with the rapid development of Taizhou economy, Taizhou

\footnotetext{
* This paper is the result of Taizhou Philosophy and Social Science Program (19GHB25).

LI Chun-feng, Master, Foreign Language School, Taizhou University, Linhai, China.
} 
Hehe (和合) Culture research has gradually been paid attention to. A large number of folk scholars consciously carry out the material sorting, connotation interpretation of Taizhou Hehe (和合) Culture and some influential folk academic works emerged as well. In recent years, the municipal party committee and government has proposed to actively create "holy land of Hehe (和合)”, highlighting the cultural self-confidence of the new era. In May 2017, the opening ceremony of Tiantai mountain culture research institute and Hehe (和合) culture research institute of Taizhou University and the first academic forum of Hehe (和合) culture and Tiantai mountain culture were held in Linhai campus of Taizhou University. In the same year, Taizhou Hehe (和合) Culture research project was successfully selected into the provincial key cultural engineering research project.

Since then, the research and inheritance of Taizhou Hehe (和合) Culture have reached a new level. Many activities related to Hehe (和合) culture, such as 100 lectures of Hehe (和合) culture, Hehe (和合) culture festival, and Hehe (和合) academy have also been vigorously carried out. However, a search on CNKI for “Taizhou Hehe (和合) Culture” and “Hanshan (寒山) poetry” showed 72 search results from 2003 to 2018, 11 of which were related to Taizhou Hehe (和合) Culture, and the rest were all about Hanshan (寒山) poetry of Taizhou Hehe (和合) Culture. Among these literatures, only six discuss translation and publicity. Thus it can be seen that there is still a lot of room for theoretical research and practical exploration of Taizhou Hehe (和合) Cultural translation and introduction.

Foreign studies on Taizhou Hehe (和合) Culture, especially in Japan and the United States, started earlier, but their scope was limited to Hanshan (寒山) poems of Taizhou “Two Saints of Hehe (和合)”. From the 1930s to the end of 1980s, Japanese scholars began to study Hanshan (寒山)'s poetry, and published more than 10 kinds of Hanshan (寒山)'s poetry and related works and interpretations in 50 years (Zigui, 2004, p. 25). American scholars began to pay attention to Hanshan (寒山)'s poems in 1954 when the Sinologist Arthur Waley published 27 transliterated Hanshan (寒山)'s poems in the magazine Encounter. In 1958, American poet Gary Snyder published 24 transliterated Hanshan (寒山) poems in the autumn Issue of Ivy Review, Volume 2, Issue 6. In the 1960s and 1970s, Hanshan (寒山) poems translated by Snyder were included in the textbooks of American universities and in the important Chinese literature collections translated from English (Zhong, 2006, p. 269). In 1982, famous Sinologist Burton Watson translated 126 Hanshan (寒山) poems, 100 poems of the Tang dynasty poet Hanshan (寒山). Although many Japanese and American researchers have paid attention to Hanshan (寒山) Zen Buddhism, they have not paid much attention to and analyzed the southern sect of Taizhou Taoism and the great Confucian Zhu Xi (朱喜), part of Taizhou Hehe (和合) Culture. Meanwhile, the rest of the world knows little about Taizhou Hehe (和合) Culture.

To sum up, although Taizhou Hehe (和合) Culture research has entered its peak period, its external translation and publicity has just started, and there is urgent room for improvement. First, although the translation of Hanshan (寒山) poems has made positive attempts to translate and introduce Taizhou Hehe (和合) Culture, few texts have systematically and comprehensively translated and introduced Taizhou Hehe (和合) Culture. Second, the existing translation of Taizhou Hehe (和合) Culture is mostly found in tourist attractions, tourism websites, public signs, publicity brochures, etc., and the translation is hard to be understood and accepted by foreign audiences, thus they cannot appreciate the charm of Taizhou Hehe (和合) Culture. Third, there is no local English website to introduce Taizhou Hehe (和合) Culture, let alone the foreign language printed materials for people to read. It is no exaggeration to say that Taizhou Hehe (和合) Culture is still dwelling narrowness in China, and has not been out to the world. This status quo is far from Taizhou's city 
orientation of “a city with mountains, seas and water, a holy land with Hehe (和合) culture” and the strategy of Chinese culture "going out" advocated by the country.

\section{The Translation and Introduction Strategy of Taizhou Hehe (和合) Culture}

The purpose of translation and introduction of Taizhou Hehe (和合) Culture is to let Taizhou go to the world, let the world know Taizhou, to carry forward Taizhou's unique regional culture and deepen Taizhou's internationalization and opening up to the outside world.

\section{Strengthening Organizational Leadership and Optimizing the Translation Environment}

Depending on the government departments to issue the policy of Hehe (和合) culture translation and introduction. The main body of translation and introduction of Taizhou Hehe (和合) Culture is diversified, including the departments of Taizhou municipal party committee and government, such as publicity department, information office, cultural tourism bureau, tourism company, scenic spot management department, and interpreter. The leading role of government departments should be given full play in the translation and introduction of Taizhou Hehe (和合) Culture, and in planning the “going out” of Taizhou Hehe (和合) Culture as a regional development strategy. Municipal publicity department, information office unite other cultural tourism bureau to formulate the corresponding standards and policies to regulate the translation and introduction of Taizhou Hehe (和合) Culture. They should establish systems and mechanisms for talent guarantee, information consultation, and job responsibility, and regularly organize propagandizing, study and review the implementation of major cultural "going global" projects to ensure the plan progress smoothly. Tourism companies and scenic spot management departments should actively participate in the translation and transmission of Taizhou Hehe (和合) Culture to improve the awareness and ability of the staff. Translators and proofreaders should improve their theoretical level and translation ability, enhance their sense of responsibility, and strictly control the quality of translation so that the connotation of Taizhou Hehe (和合) Culture can be transmitted to foreign audiences accurately and effectively.

Creating the platform of the translation of Hehe (和合) culture on the basis of universities and research institutions. The construction of talent team is the key and guarantee of "going out" of Taizhou Hehe (和合) Culture. It should be based on the actual situation of Taizhou, make full use of Taizhou University, Taizhou Vocational and Technical College, Taizhou TV University to set up relevant majors, cultivate specialized personnel in helping Taizhou Hehe (和合) Culture “going out", to ensure that the translation personnel are with high quality, strong ability, and thus make the translation work of Taizhou Hehe (和合) Culture systematic, standardized, diversified. Secondly, Taizhou Hehe (和合) Culture main unit, cultural enterprises can select their own talents and send them to professional institutions and universities for directed education. Thirdly, we should increase the introduction of domestic and foreign cultural talents to promote Taizhou Hehe (和合) Culture to faster and better “go out”.

Taking the information network as a bridge to build a translation platform in the era of big data. With the development of information technology and the advent of the era of big data, various new translation methods such as network translation, machine translation, CAI, crowd sourcing, and so on are emerging gradually. With the help of those network, we can provide foreign audiences with foreign language introduction, beautiful pictures, dynamic audio and video, discipline films, micro films, and other diversified publicity and introduction of Taizhou Hehe (和合) Culture, to ensure that foreign audiences can easily and comprehensively understand, accept, and appreciate Taizhou Hehe (和合) Culture. 


\section{Enriching the Skills of Translation and Introduction and Striving for Accurate Image}

External publicity translation should try to be vivid and accurate. According to different translation contents, various translation strategies can be adopted. The most famous and controversial translation strategies are domesticating translation and foreignizing translation. Translation scholars and cultural scholars have been heatedly arguing about the merits of domestication and foreignization a lot. Domestication means that translators should not only think about the original text and its author, but also express the content and style of the original text accurately and vividly. At the same time, the translator should also think about the readers of the translated text, and the translated text should be as close to the readers as possible, so as to facilitate their understanding and acceptance. In order to retain the cultural connotation of the source language to the maximum extent, the foreignizing translation law does not necessarily follow the grammatical rules of the target language but can ensure the cultural connotation of the original text not being lost, so it is the preferred translation strategy for the output of national culture. Lawrence Venuti, an American translator, launched a fierce attack on domestication from the perspective of post-colonial translation. On the one hand, domestication ignores the translator's position and function; in addition, although domestication is helpful to eliminate the cultural differences in translation and maintain the smoothness of the translated language, it is a cultural compromise strategy and sometimes misleads the target language readers. This paper thinks that the translation and introduction of Taizhou Hehe (和合) Culture should adopt different translation strategies according to the actual situation.

First, the translation of the classics of Taizhou Hehe (和合) Culture is aimed at the research audience with a high level of education, so the foreignization translation strategy should be adopted, and at the same time supplemented by annotated mode, which is helpful to maintain China's inherent cultural identity and promote the integration of eastern and western cultures. Second, research on the translation of Taizhou Hehe (和合) Cultural works is generally aimed at the general audiences who want to understand Chinese culture. In order to facilitate them to understand the contents of the translated text and ensure that the translated text is more readable, domestication translation strategy should be adopted to weaken the cultural differences between the source language and the target language. Third, Taizhou Hehe (和合) Culture and art design translation, involve paper cutting, patterns, and calligraphy and other cultural elements. These cultural elements are not only extensive in content, but also profound in meaning, so in the process of translation, supplemented by illustrations to explain to the audience, we can better publicize the connotation of Hehe (和合) culture.

\section{Improving the Evaluation System to Ensure Efficient Translation and Introduction}

Taizhou Hehe (和合) Culture translation is translated from Chinese into English, Japanese, Korean, and other languages; the translation effect will directly impact the international communication effect of Taizhou Hehe (和合) Culture. In order to improve the translation quality of Taizhou Hehe (和合) Culture and the effectiveness of international communication, the evaluation system of translation effect should be constructed based on the foreign audiences' feelings and reactions to the translation of Taizhou Hehe (和合) Culture. The translation of Taizhou Hehe (和合) Culture should first be reviewed by domestic and foreign experts organized by the competent departments, and then be published by the media. At the same time, opinions and suggestions of foreign audiences should be solicited through interviews, questionnaires, etc., constantly modified and improved according to the feedback of audiences, and the translated content should be regularly evaluated and updated. 


\section{Conclusion}

As is said above, the purpose of translation and introduction of Taizhou Hehe (和合) Culture is to let Taizhou go to the world, let the world know Taizhou, to carry forward Taizhou's unique regional culture and deepen Taizhou's internationalization and opening up to the outside world. In order to help Taizhou Hehe (和 合) Culture go out, we should depend on Taizhou government departments to take the lead, to build a platform of “going out”, and establish multimodal database of Taizhou Hehe (和合) Culture. Through the foreignization and domestication translation method, Taizhou Hehe (和合) Culture can spread effectively, thus promote the interaction of Taizhou Hehe (和合) Culture and the world culture, the concept of harmony and the construction of a harmonious world.

\section{References}

蔡方鹿. (1997). 中华和合文化研究及其时代意义. 社会科学研究, (6), 67-74.

高正. (2016). “一带一路”战略下外宣翻译策略研究. 北华大学学报, (6), 18-12.

郭建中. (1998). 翻译中的文化因素“异化和归化”. 外国语, (2), 13-20.

梁岩. (2010). 中国文化外宣研究. 北京: 中国传媒大学出版社.

刘泽权 \& 张丽. (2009). 异化之异化: 韦努蒂理论再批评. 外语研究, (3), 79.

装禾敏. (2014). 典籍英译与中国文化走出去. 出版发行研究, (7), 81-83.

田晨旭. (2018). 毫文化译介及“走出去”的路径选择. 黑龙江工业学院学报, (12), 107-111.

袁晓宁. (2005). 外宣英译的策略及其理据. 中国翻译, (1), 75-78.

钟玲. (2006). 史耐德与中国文化. 北京: 首都师范大学出版社.

子规. (2004). 中国的寒山与美国的冷山. 文史杂志, (6), 24-30. 